\title{
Exploring the role of the ICU nurse in the antimicrobial stewardship team at a private hospital in KwaZulu-Natal, South Africa
}

\author{
J Rout, RN, MN; P Brysiewicz, PhD \\ School of Nursing and Public Health, College of Health Sciences, University of KwaZulu-Natal, Durban, South Africa
}

Corresponding author: J Rout (joanrout@worldonline.co.za)

\begin{abstract}
Background. Care of the critically ill patient has become increasingly challenging, with a rising incidence of resistant pathogens resulting in the ineffectiveness of many antibiotics. Severe infection is associated with prolonged intensive care unit (ICU) length of stay, and increased morbidity, mortality, and healthcare costs. Antimicrobial stewardship (AMS) aims to prevent resistance and protect patients and the wider community by promoting correct antimicrobial use. The current AMS literature has failed to describe the role of the ICU nurse in this important initiative.

Objective. To explore the perceptions of AMS team members regarding the role of the ICU nurse in the AMS team.

Methods. Using a qualitative research approach, purposive sampling was used to identify participants in an ICU. Semi-structured interviews were conducted with 15 participants, including ICU shift-leader nurses, nursing management, surgeons, anaesthetists, physicians, microbiologists and pharmacists. Data were analysed and categorised using content analysis. The study was conducted in a general ICU in the private healthcare sector in KwaZulu-Natal, South Africa.

Results. Participants representing various disciplines of the AMS team felt that the role of the ICU nurse within the team was an important part of the AMS programme. Four categories that emerged from the data are discussed: organisational, advocacy, clinical and collaborative roles.

Conclusion. The role of the ICU nurse was found to be essential to the success of AMS in the ICU. These findings provide implications for practice, which, if recognised and supported by all healthcare stakeholders from ICU and hospital management, could improve AMS in this acute care area.
\end{abstract}

S Afr J Crit Care 2017;33(2):46-50. DOI:10.7196/SAJCC.2017.v33i2.331

Globally, antimicrobial resistance has become one of the most important issues facing critical care. Resistance to antibiotics is escalating owing to overuse of antibiotics in the community and in hospitals, with increasing use of broad-spectrum antibiotics and delayed de-escalation practices. ${ }^{[1]}$ Decreasing availability of antimicrobial therapy to treat complicated infections is attributed to the growing ineffectiveness of these medications due to increasing resistance, and the move of the pharmaceutical industry away from research and development of these products. ${ }^{[2]}$ Therefore, it is becoming more difficult to manage infections in the very ill patient, resulting in prolonged intensive care unit (ICU) stay and increased mortality. ${ }^{[3]}$

Antimicrobial stewardship (AMS) has developed as a proactive healthcare initiative to reduce antibiotic-selective pressure by promoting appropriate use of antimicrobial therapy and effective infection control. This is especially important in an ICU, which has the highest proportion of patients vulnerable to infection and uses the most antibiotics in the hospital setting. ${ }^{[4]}$ Research into the prevalence of infection in private and public ICUs in South Africa (SA) found that, in the private ICU sector, an inappropriate antibiotic was prescribed in $60.8 \%$ of patients, duration of administering an antibiotic was inappropriate in $81.7 \%$ of patients, and de-escalation was used in only $19.7 \%$ of patients. The authors attributed these figures to poor prescribing practices in this sector. ${ }^{[5]}$

Infection control has become a large part of nursing responsibilities in the ICU arena, but the nurse does not yet have clear responsibilities regarding antimicrobial therapy, and it appears that the role and skills required of the nurse in AMS have not been adequately explored. ${ }^{[6,7]}$

\section{Objective}

The purpose of this qualitative study was to explore AMS team members' perceptions of the role of the ICU nurse within the AMS team.

\section{Methods}

A qualitative research approach was chosen, as it allows in-depth examination of a subject and is open to the multiple realities of multidisciplinary participants. ${ }^{[8]}$

\section{Setting}

This study was conducted in the general adult 20-bed ICU/high-care unit in a 200-bed private hospital in KwaZulu-Natal, SA, where a new AMS programme was being implemented by nursing management.

\section{Participants}

After institutional ethical review and gate-keeper permission, purposive sampling identified 9 nursing and 8 non-nursing members of the ICU team most likely able to answer the research question; they were invited to participate ${ }^{[8]}$ Inclusion criteria for nursing participants were as follows: nurses registered with the SA Nursing Council; those involved in setting up the AMS programme; or ICU shift leaders conducting daily AMS rounds. Specialised ICU training was not a criterion. Non-nursing participants were from the disciplines of medicine, surgery, anaesthesiology, pharmacy and microbiology and were included if they prescribed antibiotics/antifungals to patients, dispensed this treatment or were consulted regarding laboratory findings in the ICU. 


\section{Data collection process}

After informed consent by participants and assurance of anonymity and confidentiality, data were collected during 3 weeks in August 2014. Semi-structured interviews were held in a private room in the hospital and were chosen to allow flexibility in the interview process. Core questions were used as a basis to facilitate discussion of the topic. These predetermined open-ended questions provided an initial focus of attention for both the researcher (JR) and the participants, and issues raised by the participants in the interviews allowed the researcher to explore a wide range of topics, further developing these in subsequent interviews. Participants were encouraged to talk frankly and were assured that no responses were viewed as being incorrect. A verbatim, typed transcript of the recorded interview was given to each participant to check for accuracy and to make additional comments if necessary.

\section{Data analysis}

Interview transcripts were subjected to content analysis to identify categories from the data, guided by the purpose of the study. A process of interpretation was carried out by familiarisation with the text, examining each participant's contribution and paraphrasing and condensing the content of the text (Table 1) to determine codes and categories. ${ }^{[9]}$

\section{Rigour}

Academic rigour was to be met by the required qualitative criteria of trustworthiness, credibility, transferability, dependability and confirmability. ${ }^{[10]}$ Credibility was assured by researcher familiarity with the culture of the study setting as an ICU nurse, request for participant honesty, review of transcript analysis by a more experienced researcher and returning transcript analysis to participants following initial analysis to validate the findings. Transferability was assured by providing a detailed context for the study and a comprehensive description of phenomena being studied. For dependability, a detailed methodology was described by the researchers. Confirmability was assured by record keeping and the use of multidisciplinary participant viewpoints to provide a coherent, deeper perspective.

\section{Ethical approval}

After approval by the Ethics Committee of the University of KwaZuluNatal (ref. no. BE281/14) and hospital management, participants were invited to take part in the study. Information was given to the participants prior to written consent, which emphasised the right to decline participation in or withdraw from the study at any stage. Permission was obtained to record the interviews. Participants were invited to choose pseudonyms to ensure anonymity and confidentiality.

\section{Results}

Fifteen participants from six different disciplines agreed to take part: 8 nurses and 7 non-nurses. Two potential participants declined to participate in the study - 1 nursing and 1 non-nursing. Two nursingmanagement participants described their experiences of starting the AMS programme. Six ICU nurse shift leaders discussed how they conducted daily AMS rounds and communicated with other members of the ICU team. Two surgeons, 2 physicians and 1 anaesthetist shared their perceptions of working with nurses during the daily routine of ward rounds and treatment changes. A microbiologist and pharmacist discussed their experiences of working with both nurses and prescribers.

Four categories emerged from the content analysis, i.e. organisational, advocacy, clinical and collaborative roles.

\section{Organisational role}

Nursing participants explained that the AMS programme in the ICU was a nursing initiative designed to monitor antibiotic/antifungal use in the unit. There was minimal knowledge or experience of how to set up or run this initiative. Of the 15 participants who took part in the study, only 2 of them - 1 nursing and 1 non-nursing - had previously been part of an AMS programme and only the non-nursing participant had gained experience in SA:

'Initially there was absolutely no awareness of antibiotic stewardship among the registered nurses that started. And I think that this was maybe because they were all from different hospitals and maybe they didn't really know about ... and it was also a new thing, I think ... and so we had to explain to them what we're doing and get their buy in into it.' (Nursing participant)

Nursing participants described how daily shift-leader rounds were performed each morning in the ICU to monitor Best Care ... Always! (BCA) bundle compliance, documentation of antibiotic treatment, tracking of laboratory results, antibiograms and any changes in doctors' instructions. BCA is an SA national campaign to reduce healthcareassociated infections and introduce AMS to the SA setting. Documentation of laboratory results, invasive 'line days' and duration of antimicrobial therapy was routinely carried out by the floor nurse allocated to care for

Table 1. Example of content analysis coding and categorisation

Meaning units
'Initially there was absolutely no awareness of antibiotic stewardship among
the registered nurses.'

the registered nurses.'

... we had to explain to them what we're doing and get their buy in into it.'

'Because the doctors can miss the results if we don't draw it to their attention. And that includes antibiotic days.'

'... and the other thing is washing hands. That is very, very important, to prevent Need for diligent cross infection between patients.'

‘... we try to have a weekly meeting ... It doesn't always happen, but during that meeting we do a case discussion, so we will talk about that patient, the clinical picture of the patient, together with the laboratory results, what antibiotic the patient is on .... ?

\begin{tabular}{lll} 
Condensations & Codes & Category \\
\hline $\begin{array}{l}\text { No awareness } \\
\text { of antibiotic } \\
\text { stewardship }\end{array}$ & Educating & Organisational role \\
$\begin{array}{l}\text { Explain to get } \\
\text { buy-in } \\
\text { Alerting doctors }\end{array}$ & Setting up & Organisational role \\
$\begin{array}{l}\text { Need for diligent } \\
\text { practice }\end{array}$ & $\begin{array}{l}\text { Infection } \\
\text { prevention and } \\
\text { control } \\
\text { Communication }\end{array}$ & Collaborative role \\
$\begin{array}{l}\text { Discussing the } \\
\text { condition of ICU } \\
\text { patients }\end{array}$ & Clinical role \\
\hline
\end{tabular}


a specific patient during a 12-hour shift. Nursing participants viewed the monitoring of line days as an important daily task and invasive lines were removed when no longer indicated. Participants explained:

'What I expect of them is to do the daily rounds, preferably in the morning after the doctor rounds. Just what we normally do is we check your patient's infection markers ... if it was done that day. You write all your results in and then you also write the antibiotic. The name, the dose, the strength and how often it gets given ... I want them to monitor all that.' (Nursing participant)

'Very important, they usually help me ... with keeping the book. They will tell me how long the lines have been in. They will alert me on ... "Listen this CVP's been in for two weeks and the wound site looks red ... ." In other words, they would make sure I don't overlook the things, especially with long-admitted patients.' (Non-nursing participant)

\section{Advocacy role}

Nursing and non-nursing participants felt strongly that patients should be given the correct antimicrobial treatment for an infection and that the ICU nurse had a responsibility to promote the interests of the patient:

'Talking on behalf of the patient ... Making the initiative to actually do something for them ... you know ... for the patient. So, we're acting on behalf of the patient, telling the doctor. Look ... this is what bug the patient's got and this is what antibiotic can be given.' (Nursing participant)

'The best thing about the intensive care nurse is that she has all the information in that file at that point ... she has the culture results ... she sees the doctor every day. She would know when the dose is given, when it was not given ... the duration.' (Non-nursing participant)

De-escalation was raised as a key aspect of correct antimicrobial management. Nursing participants felt that an important part of their role was to monitor the duration of antimicrobial therapy and alert specialists to 'antibiotic days' to contribute to the de-escalation process. Non-nursing participants agreed:

'Because the doctors can miss the results if we don't draw it to their attention. And that includes antibiotic days. Because you will see patients on day 8 , day 9 , day 14 , and that's when we say to the doctor ... "This antibiotic we've been doing it for 14 days" ... and "Oh, maybe we need to look at stopping it, maybe we need to look at changing it" ...? (Nursing participant)

'So, the nurses' function is to make sure that the correct antibiotic has been given and it has been correctly given. They can also monitor that this patient has been on it for 7 days and this patient has not improved and they can discuss with the doctor'. (Non-nursing participant)

\section{Clinical role}

Nursing and non-nursing participants agreed that the nurses' ongoing monitoring of the critically ill patient was essential to identify early signs of infection:

'We monitor the patient's temperature and specific bloods like the PCT, the white cells and the CRP and also what antibiotic and how many days they've been on that antibiotic ... what dosage and what duration ... ? (Nursing participant)

'Very important role ... I mean the intensive care nurse is there, you know, all the time. They are monitoring the trends ... they can pick up the little subtle things that happen in change of condition. We're there twice a day for a short period of time. The ICU nurse is vital in the care of the patient ... it's a massive responsibility.' (Non-nursing participant)
Nursing and non-nursing participants agreed that infection control should be properly managed in the ICU to minimise healthcare-associated infections, cross-infection and environmental resistance:

'... and the other thing is washing hands. That is very, very important, to prevent cross infection between patients.' (Nursing participant) 'Still universal precautions are very important ... and they are the ones doing it. Fortunately, they work with one patient only, but I can imagine if you quickly have to do something for the patient next door, you actually need to unscrub, unglove, wash and go to the next one. And that is important because that is how antibiotic resistance spreads as well.' (Non-nursing participant)

\section{Collaborative role}

Nursing participants described daily ward rounds during which doctors reviewed antimicrobial treatment at the bedside of their patient and gave instructions to the floor nurse allocated to care for that patient for the day. The role of the ICU nurse in communicating information to doctors was identified by non-nursing participants as an important part of stewardship:

'So, for communication they are important, for source control they are important, for making sure the actual antibiotic is given they are important ... because, let's be honest, they do the actual work.' (Nonnursing participant)

Nursing participants reported a hierarchical nursing structure in the ICU, with the floor nurse working closely with the shift nurse throughout the day. Frequent communication took place with regard to the condition of patients, infective markers and the need for antimicrobial therapy. Shift leaders would then discuss developments with the ICU unit manager who was in regular contact with the infection control co-ordinator:

'The floor nurse, who is looking after the patient ... she's got to make sure that the records on her charts are up-to-date and that the lab records are in good nick in the file. So, it starts from the floor nurse. Then it goes to the team leader on the floor. She does a round ... on all the patients and she updates, you know, picks up ... any problems ... if there's a problem with the patient. And then it goes to the manager of the unit.' (Nursing participant)

Nursing participants described contact with microbiologists during the weekly telecommunication AMS meetings:

'... we try to have a weekly meeting ... It doesn't always happen, but during that meeting we do a case discussion, so we will talk about that patient, the clinical picture of the patient, together with the laboratory results, what antibiotic the patient is on ... what other therapies involved with the patient. And we talk about infection control practices as well, like isolation of the patient and hand washing and so forth ... ? (Nursing participant)

\section{Discussion}

Findings showed that participants representing various disciplines of the AMS team felt that the role of the ICU nurse in the team was an essential part of the AMS programme. The value of the ICU nurse has been identified as: organising shift-leader AMS rounds and diligent record keeping for data surveillance; advocating for antimicrobial treatment that is in the best interests of the patient; administering antimicrobial therapy correctly, preventing healthcare-associated infections; and ongoing clinical monitoring of the ill patient for signs of infection and documenting; and communicating these findings to other members of the AMS team. 


\section{Organisational role}

At the time of the study, the AMS programme in the ICU had been in progress for 2 years. Nursing management started this soon after the study hospital had opened in response to a growing awareness of the need to manage ICU environments optimally, and provide safe care for patients. ${ }^{[4]}$ Small community hospitals use antimicrobial therapy more frequently than large academic medical centres and are less likely to have AMS programmes in the acute-care setting. ${ }^{[1]}$ The early detection of pathogens, appropriate use of antibiotics and reduction of healthcare-associated infections are essential in the care of the critically ill patient, and all ICUs should have AMS programmes that aim to ensure prompt, effective treatment. ${ }^{[12]}$ All the nursing participants felt that it was an important qualitycontrol initiative, although this type of intervention was new to them. Only two participants had previous experience of AMS.

Nursing participants viewed the organisational role of the ICU nurse as setting up the AMS programme in the ICU according to the Centers for Disease Control and Prevention (CDC) guidelines, daily monitoring of patient records during shift-leader rounds to ensure BCA bundle compliance, documenting of antibiotic/antifungal treatment, tracking of laboratory results and antibiograms, and noting any changes in doctors' instructions. Audits, compliance and feedback are important in an AMS programme, ${ }^{[13]}$ and documentation from the shift-leaders' rounds was also used for the collection of data by the hospital's infection prevention and control nurse for long-term analysis of nursing care, antimicrobial usage, and resistance profiles in the ICU. Non-nursing participants identified management of laboratory results as the main organisational aspect of the nurse's role in AMS.

\section{Advocacy role}

There is a growing awareness among health professionals of the enormity of the challenges that face healthcare in the 21st century. Nurses should be an important part of AMS, and monitoring of antibiotic/ antifungal use should be done by hospital pharmacists, microbiologists, doctors and nurses. Nursing and non-nursing participants felt strongly that patients should be administered the correct antimicrobial treatment for an infection and that they had a responsibility to promote the interests of the patient by reminding specialists of 'antibiotic days' to facilitate de-escalation. All participants interviewed showed a good understanding of AMS and that the objective is to promote the appropriate use of antimicrobial therapy, with the correct choice of antibiotic/ antifungal, the correct dose and the prompt use of antibiograms to direct de-escalation. ${ }^{[14]}$

\section{Clinical role}

An important part of AMS is to minimise bacterial resistance by implementing effective infection control practices ${ }^{[1]}$ and monitoring infections, infective markers and invasive lines. Nursing and nonnursing participants perceived the ICU nurse's clinical responsibilities as the monitoring of changes in the condition of the ill patient to identify early signs of infection, specimen collection, correct administration of antibiotics/antifungals, and adherence to infection control protocols by all ICU staff and visitors to minimise healthcare-associated infections, cross-infection, and environmental resistance. Stewardship monitoring was added to daily ICU shift rounds in this ICU, and BCA bundles were introduced to ensure evidence-based patient care ${ }^{[6]}$ AMS is promoted as the fifth pillar, or bundle, of the BCA campaign in SA. ${ }^{[15]}$

\section{Collaborative role}

Intercepting a verbal or written prescription, discussing it with the doctor and confirming that it is the correct drug based on microbiology results contribute to patient safety. ${ }^{[16]}$ Nursing and non-nursing participants thought that nurses working in an ICU in the private healthcare sector in SA have a very important role to play, as they are at the patients' bedside 24 hours a day, whereas medical specialists are not able to be present in the ICU at all times. Nursing and non-nursing participants identified the collaborative role of the ICU nurse in AMS as conducting shiftleader stewardship rounds, participating in doctors' bedside rounds and communicating telephonically with doctors. Microbiologists from a private laboratory were instrumental in providing guidance and support for the AMS programme and individually to doctors, and participated actively in the weekly telecommunication AMS meetings. The contact with microbiologists allowed very important analysis of patient care and of the treatment plan, and facilitated surveillance-data collection by the infection control nursing co-ordinator. ${ }^{[17]}$

\section{Study limitations}

The study findings are contextual, specific to the study hospital and have limited generalisability owing to non-random selection in a single ICU.

\section{Conclusion}

The nurse had a strong organisational role in this nurse-driven AMS programme, which was set up as a quality-improvement initiative in a new 'clean' hospital, with the aim of adhering to international AMS guidelines by promoting these principles among all stakeholders, and careful record keeping to assist with antimicrobial monitoring and pathogen surveillance. There was awareness of the advocacy role of the nurse in reminding prescribers of the duration of antimicrobial therapy, and alerting them to antibiograms. The clinical role of the nurse in monitoring infection and infection control was emphasised in this ICU. Nurse interaction with other members of the AMS team was seen as vital to effective communication.

\section{Recommendations}

A sound understanding of the principles and objectives of AMS is vital for ICU nurses to advocate for the process of de-escalation of antimicrobial therapy. ICU nurses should actively participate in AMS, according to World Health Organization and CDC guidelines. ICU nurses are advised to follow infection control bundle recommendations, such as those of the BCA initiative. Diligent monitoring of the critically ill patient is needed to identify early signs of infection, and ICU nurses should be able to effectively communicate changes in the patient's condition to the relevant member of the healthcare team.

Acknowledgements. We wish to acknowledge Dr J de Beer for her assistance in conceptualising the study.

Author contributions. JR: collected the data; JR and PB: analysed the data, drafted the manuscript and approved it for submission.

Funding. None.

Conflicts of interest. None.

1. Mendelson M, Whitelaw A, Nicol M, et al. Wake up, South Africa! The antibiotic 'horse' has bolted. S Afr Med J 2012;102(7):607-608. https://doi.org/10.7196/SAMJ.5759

2. Gould I, Bal A. New antibiotic agents in the pipeline and how they can help overcome microbia resistance. Virulence 2013;4(2):185-191. https://doi.org/10.4161/viru.22507

3. Deege M, Paterson D. Reducing the development of antibiotic resistance in critical care units. Curr Pharm Biotechnol 2011;12(12):2062-2069. https://doi.org/10.2174/138920111798808301

4. Kaki R, Elligsen M, Walker S, et al. Impact of antimicrobial stewardship in critical care: A systematic review. J Antimicrob Chemother 2011;66(6):1223-1230. https://doi.org/10.1093/jac/dkr137

5. Paruk F, Richards G, Scribante J, et al. Antibiotic prescription practices and their relationship to outcome in South African intensive care units: Findings of the Prevalence of Infection in South African Intensive Care Units (PISA) study. S Afr Med J 2012;102(7):613-616. https://doi.org/10.7196/SAMJ.5833 
6. Ziady L. Antibiotic stewardship: A nut without a definite shell to crack. Prof Nurs Today 2012;16(5):12-14. 7. Edwards R, Drumright L, Kiernan M, et al. Covering more territory to fight resistance: Considering nurses' role in antimicrobial stewardship. J Infect Prevent 2011;12(1):6-19. https://doi. org $/ 10.1177 / 1757177410389627$

8. Erlingsson C, Brysiewicz P. Orientation among multiple truths: An introduction to qualitative research. Afr J Emerg Med 2013;3(2):92-99. https://doi.org/10.1016/j.afjem.2012.04.005

9. Elo S, Kyngas H. The qualitative content analysis process. J Adv Nurs 2008;62(1):107-115. https:// doi.org/10.1111/j.1365-2648.2007.04569.x

10. Guba E. Criteria for assessing the trustworthiness of naturalistic inquiries. Educ Commun Technol 1981;29(2):75-91.

11. Storey D, Patel P, Nguyen A, et al. Implementation of an antimicrobial stewardship program on the medical-surgical service of a 100-bed community hospital. Antimicrob Resist Infect Control 2012;1(32):1-8. https://doi.org/10.1186/2047-2994-1-32

12. Kollef M, Micek S. Antimicrobial stewardship programs: Mandatory for all ICUs. Crit Care 2012;16(179):1-2. https://doi.org/10.1186/cc11853
13. Du Toit B. The role of the infection control practitioner in antimicrobial stewardship. Mediclinic Nursing 2012:26-27.

14. Chunnilall D, Peer A, Naidoo I, et al. An evaluation of antibiotic prescribing patterns in adult intensive care units in a private hospital in KwaZulu-Natal. S Afr J Infect Dis 2015;30(1):17-22. https://doi.org/10.1080/23120053.2015.1103956

15. Kantor G, van den Bergh D, Brink A. Best Care, Always! S Afr J Epidemiol Infect 2011;26(1):3-5 https://doi.org/10.1080/10158782.2011.11441411

16. Dickson G, Flynn L. Nurses' clinical reasoning: Processes and practices of medication safety. Qual Health Res 2012;22(1):3-16. https://doi.org/10.1111/j.1475-6773

17. Charani E, Cooke J, Alison A. Antibiotic stewardship programmes - what's missing? J Antimicrob Chemother 2010;65(11):2275-2277. https://doi.org/10.1093/jac/dkq357

Accepted 28 August 2017. 